\title{
Posterior cruciate ligament reconstruction with "all-inside" technique: a technical note
}

\author{
Corrado Bait \\ Matteo Denti \\ Emanuele Prospero \\ Alessandro Quaglia \\ Antonio Orgiani \\ Piero Volpi
}

Knee Surgery and Sports Traumatology Unit, Humanitas Research Hospital, Rozzano, Milano, Italy

Corresponding author:

Corrado Bait

Knee Surgery and Sports Traumatology Unit, Humanitas Research Hospital

Via Manzoni 56

20089 Rozzano, Milano, Italy

E-mail: corrado.bait@humanitas.it

\section{Summary}

Introduction: posterior cruciate ligament (PCL) injuries are an increasingly recognized cause of knee instability in the practice of orthopaedic surgery and sports medicine. Clinical interest in these injuries has been increasing over the last several decades as knowledge and understanding of the biomechanical consequences and surgical reconstruction options have progressed. These injuries can be extremely challenging for the treating physician as substantial controversy exists regarding the optimal management of this problem. There has also been increasing interest and recognition of the importance of secondary stabilizing structures, including the posterolateral and posteromedial corner injuries as well as the issues with malalignment that must also be addressed at the time of PCL surgery to optimize results. Thanks to the continuous research for a correct anatomical placement and new systems of fixation, we can now perform the tibial and femoral bone tunnel more easily and safely by retrograde out-in approach with a special "drill-pin".

Conclusion: this technique provides a graduated precise execution of the tunnels. New methods were also developed to determine the so-called "second-generation cortical suspensory fixations" that have the feature of being "Adjustable": shortens implant by pulling on strands to allow cinching graft passing and tensioning button to regulate their length after fixation and then to create tension in the new graft, once introduced into the joint.

KEY WORDS: knee arthroplasty, implant position, unicompartmental, patient specific, alignment.

\section{Introduction}

Posterior cruciate ligament $(\mathrm{PCL})$ injuries are an increasingly recognized cause of knee instability in the practice of orthopaedic surgery and sports medicine. Clinical interest in these injuries has been increasing over the last several decades as knowledge and understanding of the biomechanical consequences and surgical reconstruction options have progressed. These injuries can be extremely challenging for the treating physician as substantial controversy exists regarding the optimal management of this problem. These controversies include the benefits of operative $v s$ nonoperative treatment, acute vs delayed repair, single- $v s$ double-bundle reconstructions, tibial in lay $v s$ transtibial tunnel reconstruction, femoral outside-in $v s$ inside-out drilling, and graft choices, including the use of autograft $v s$ allograft tissue ${ }^{1-5}$. There has also been increasing interest and recognition of the importance of secondary stabilizing structures, including the posterolateral and posteromedial corner injuries as well as the issues with malalignment that must also be addressed at the time of PCL surgery to optimize results. Posterior cruciate ligament injuries may be classified with respect to timing (acute versus chronic) and severity of injury (isolated or combined), and both variables will directly affect the treatment and prognosis ${ }^{6}$. Much work has been done through anatomy dissections and anatomical sectioning studies and biomechanics to improve our understanding of native PCL function as well as the importance of the secondary stabilizers. The poor functional results achieved with surgical arthrotomy techniques ${ }^{7}$ led in time to take a conservative approach for PCL injuries and only in the last two decades, the data reported in the literature on severe outcomes resulting from the absence of the PCL and the recognition of the importance biomechanics of the ligament in the creation of a central pivot point strongly encouraged to re-evaluate the surgical treatment and to seek new surgical solutions ${ }^{8,9}$. 
Thanks to the continuous research for a correct anatomical placement and new systems of fixation, we can now perform the tibial and femoral bone tunnel more easily and safely by retrograde out-in approach with a special "drill-pin". This provides a graduated precise execution of the tunnels.

New methods were also developed to determine the so-called "second-generation cortical suspensory fixations" that have the feature of being "Adjustable": shortens implant by pulling on strands to allow cinching graft passing and tensioning button to regulate their length after fixation and then to create tension in the new graft, once introduced into the joint.

\section{Surgical technique}

Posterior Cruciate Ligament "all-inside" reconstruction with no-tunnel technique requires a lot of "arthroscopic experience" because anatomical position of $\mathrm{PCL}$ is more complex than $\mathrm{ACL}$, and then requires learning new graft preparation for use a new special drilling and graft fixation technique.

Semitendinosus tendon autograft generally use in this technique, the patient, under general or a spinal anaesthesia, is prepared and placed in a leg holder permitting knee movement from $0^{\circ}$ to $90-100^{\circ}$, sometimes we use autograft, in case of multiple reconstruction, and we prefer tibialis posterior or anterior tendon.

A routine arthroscopic examination is performed at the end to evaluate the PCL lesion, to confirm correct anatomic insertion and position and to treat any associated lesion, (meniscus, cartilage, etc.). The arthroscope is withdrawn, a small vertical anteromedial incision is made at the level of the pes anserinus and the Semitendinosus is harvested. When the subcutaneous tissue is separated from the sartorius fascia, the insertion point of the half-tendons is clearly visible. At this point, the entire insertion of the pes anserinus is detached to ensure a clear view of the course of the tendons defined from below. The tendon is detached using a common tendon stripper and then, after harvesting, the sartorius fascia is sutured to the original insertion point. The tendon is placed on the special graftmaster, the size and diameter are prepared suturing the graft in a loop at a specific length (generally 6-7 cm depending of at the size of the knee) including TighRope and TighRope RT (Arthrex Naples, FL) (Fig. 1).

The preparation of the graft is very important. It's mandatory that only a precise and accurate measurement can guarantee a correct intrarticular tightness.

After reintroducing the arthroscope, only the intercondylar notch is cleaned in the femoral footprint of $\mathrm{PCL}$ and then we create a postero-medial (PM) portal to introduce a service cannula $(5,5 \mathrm{~mm})$ through which we prepare a tibial footprint. It's a very important step to clean carefully the femoral and tibial footprint for this we use the second-generation drill called "Flipcutter", evolution of "Retrodrill" (Arthrex Naples, FL).

The Flipcutter drill pin measures $3.5 \mathrm{~mm}$ in diameter, allowing femoral and tibial socket creation, from 6 to 13 $\mathrm{mm}$, and also half sizes, through a small skin incision.
The Flipcutter guide pin becomes a retrograde drill by flipping a switch on the pin handle, then after socket creation with clockwise drilling and retrograde pressure, the Flipcutter is switched back into a guide pin and is removed. The flipcutter guide pin is drilled through a unique graduated tip guide with a pin sleeve and a special tibial aimer designed for PCL reconstruction to protect the posterior part of the joint capsula.

Introducing a special PCL tibial guide through the AM portal and placing the tip, under scope view from the PM portal into the posterior intercondylar fossa, between the tibial plateaus in the trapezoidal area of PCL origin (Fig. 2), the Flipcutter is drilled into the tibia and out through the posterior cortex. The guide is removed and then flipping a switch on the pin handle to create a socket with clockwise retrograde drilling until $30-35 \mathrm{~mm}$ of lenght. The Flipcutter is switched back into the guide pin and removed. After the Flipcutter is removed, the sleeve is left in place, facilitating simple and reproducible passage of graft-passing suture into the joint and out through the AM portal for later graft passage.

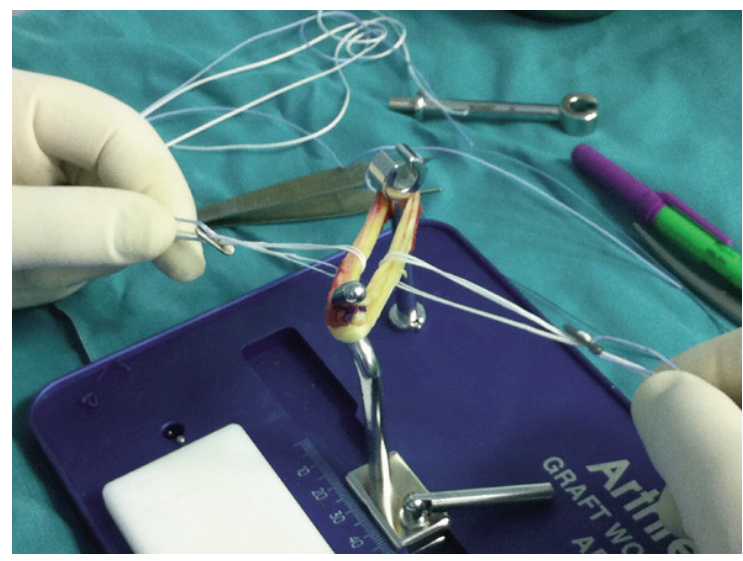

Figure 1. The preparation of the graft is a fundamental stage for this technique in that only precise and accurate measurement can guarantee a correct intrarticular tightness.

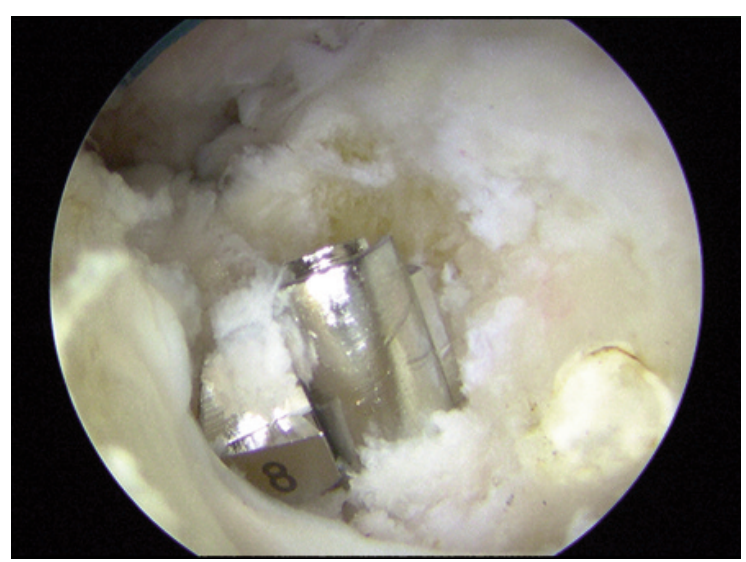

Figure 2. Arthroscopic view of tibial tunnel drilled with the Flippcutter. 
The femoral half tunnel is created out-in using a dedicated guide positioned in the femoral insertion of the PCL by AM portal (Fig. 3) in the same way that we used the Flipcutter in the tibia, until $30-35 \mathrm{~mm}$ of lenght. After drilling, the Flipcutter is removed and a suture shuttle is passed into the joint and out through the AM portal. After measuring the total length of the femoral tunnels, it is reported on the rope of the fixation device, starting from the metallic plate, and once certain that it is in the joint, the fixation device is released from the cortical.

Now we have a pair of double suture shuttles in the AM portal and it's most important to separate them clearly. We put the rope of the fixation device (TightRope) into the loop of the femur and tibial suture shuttle and introduce the graft, pulling the suture shuttle, into the joint from AM portal and out of the skin. Now with the scope in AL portal, introduce a metallic trocar through to the PM cannula to assist the graft passage in the tibia like a pulley until the metallic bar is out of the tibial cortex. A part of the graft is introduced into the tibial tunnel and then through the femoral suture shuttle, a portion of the graft is introduced until the mark on the rope as explained before. At this stage switch the scope from the AL to the AM portal while the AL portal recuperates the rope to create the tension of the graft. At this moment the femoral device is flipped out of the femoral cortex and pulled tight under scope view. Move the knee at $70^{\circ}$ of flexion in anterior drawer, and pull tight the tibial rope out of the cortex to create the correct tension of the graft under scope view (Fig. 4).

The final check and apply a knee brace with posterior support to prevent posterior drawer by gravity. The rehabilitation is not $A C L$ in reverse, but a much slower progression, continuous passive motion with Kinetec ${ }^{\circledR}$ starting from second day post-op for 4 weeks and then, remove the brace, pool exercise and bike are permitted. Return to heavy-work is at 4-6 months and allow return to sport at about 1 year.

In accordance with international standards and as required by this journal, authors declare that the study meets the ethical standards of this journal ${ }^{10}$.

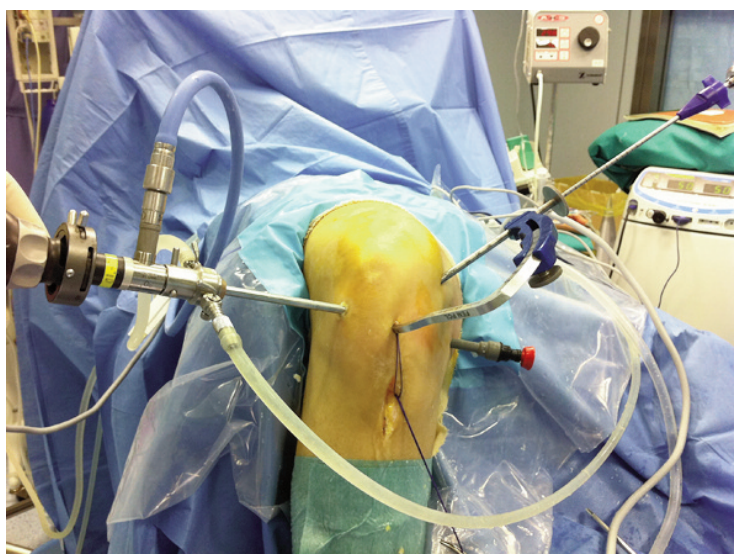

Figure 3. Dedicated guide to drill femoral tunnel.

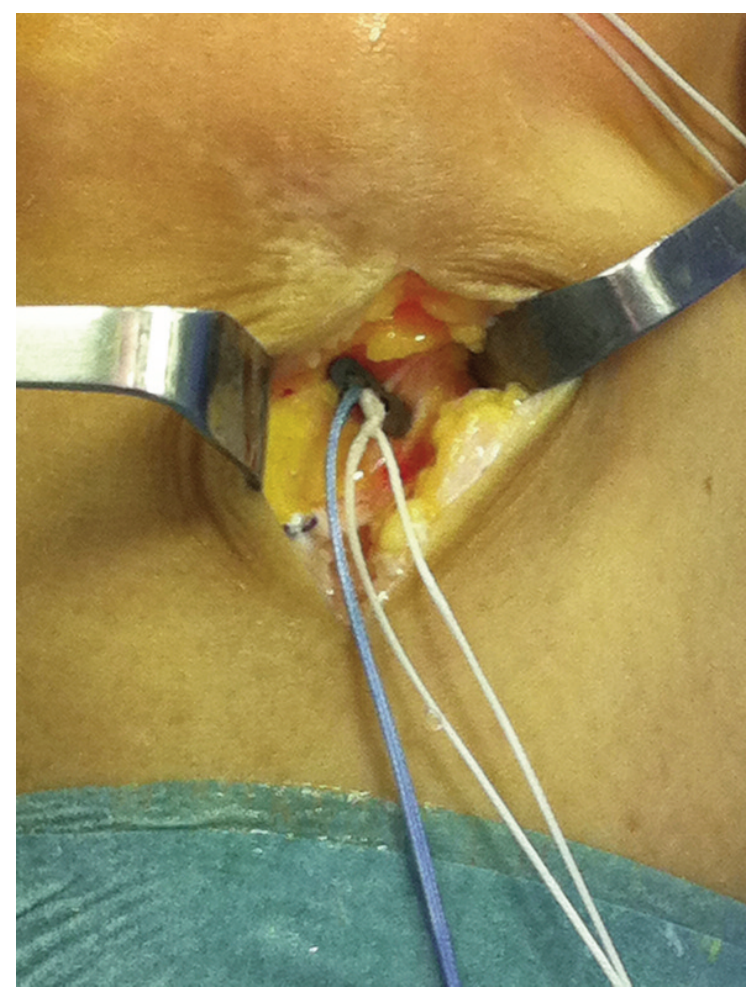

Figure 4. External tibial cortex with the metallic bar fixed.

\section{Discussion}

The reconstruction of the PCL has always been a very complex and technically difficult operation in respect to the ACL reconstruction, especially in the preparation of the tunnel or in particular the mode in which it always represents a dangerous passage so close to the vascular popliteal nerve. The all-inside technique allows, thanks to extremely precise instruments, the creation of two half tunnels in a secure and reproducible manner, without the use of the amplifier of brightness to display the position of the tibia guide wire ${ }^{11}$. A secure advantage in this technique is undoubtedly represented by the possibility to reconstruct a ligament, very important to the biomechanics of the knee, with a single autologous tendon. In fact it is sufficient to get a quadruple semitendinosus ligament size valid to replace the PCL. The traditional system to fix the PCL has always had, especially at the tibia, a specific problem of reliability for the lower bone density tibia insertion, sometimes the interference screws "sinking" in the cancellous bone ${ }^{12}$; the possibility to have a cortical fixation also at the tibia level guarantees greatly an advantage for the bone integration and tension of the graft. In addition, these new systems allow cortical fixation to tension the graft after it's fixed with relative ease. This method, in our opinion, provides a correct reconstruction of the $\mathrm{PCL}$ with the construction of anatomic bone tunnels using highly accurate and therefore reproducible instruments, in a less invasive surgery, sparing the cortical of the femur and tibia and a viable graft fixation. 


\section{References}

1. Sekiya JK, West RV, Ong BC, Irrgang JJ, Fu FH, Harner CD. Clinical Outcomes after Isolated Arthroscopic Single-Bundle Posterior Cruciate Ligament Reconstruction. Arthroscopy. 2005;21(9):1042-1050.

2. Seon JK, Song EK. Reconstruction of Isolated Posterior Cruciate Ligament Injuries: A Clinical Comparison of the Transtibial and Tibial Inlay Techniques. Arthroscopy. 2006;22:1.

3. MacGillivray JD, Stein Be, Park M, et al. Comparison of Tibial Inlay versus Transtibial Techniques for Isolated Posterior Cruciate Ligament Reconstruction: Minimum 2-Year Follow-Up. Arthroscopy. 2006;22(3):320-328.

4. Klimkiewicz JJ, Harner CD, Fu FH. Single Bundle Posterior Cruciate Ligament Reconstruction: University of Pittsburgh Approach. Operative Techniques in Sports Med. 1999;7(3):105109.

5. Goudie EB, Will EM, Keating JF. Functional Outcome Following PCL and Complex Knee Ligament Reconstruction. The Knee. 2010;17(3):230-234.

6. Chen $\mathrm{CH}$, Chen WJ, Shih $\mathrm{CH}$, Chou SW. Arthroscopic Poste- rior Cruciate Ligament Reconstruction with Quadriceps Tendon Autograft: Minimal 3 Years Follow-Up. Am J Sports Med. 2004;32(2):361-368.

7. Lipscomb AB Jr, Anderson AF, Norwig ED, Hovis WD, Brown DL. Isolated posterior cruciate ligament. Long Term results. Am J Sports Med. 1993;21(4):490-496.

8. Juergensen K, Edwards JC, Jakob RP. Positioning of the Posterior Cruciate Ligament. Knee Surg Sports Traumatol Arthrosc. 1994;2(3):133-137.

9. Rubinstein RA Jr, Shelbourne KD, McCarroll JR, VanMeter $\mathrm{CD}$, Rettig AC. The Accuracy of the Clinical Examination in the Setting of Posterior Cruciate Ligament Injuries. The American Journal of Sports Medicine. 1994;22(4):550-557.

10. Padulo J, Oliva F, Frizziero A, Maffulli N. Muscles, Ligaments and Tendons Journal. Basic principles and recommendations in clinical and field science research. MLTJ. 2013;4:250-252.

11. Don Johnson. Posterior Cruciate Ligament Injuries: My Approach. Operative Techniques Sports Med. 2009;17:167-174.

12. Harner CD, Hoher J. Evaluation and Treatment of Posterior Cruciate Ligament Injuries. Am J Sports Med. 1998;26(3):471482. 